\title{
Chromospheric and Coronal Heating Due to the Radiation and Collisional Damping of Fast Magnetosonic Surface Waves
}

\author{
W. Sahyouni*, Zh. Kiss'ovski, and I. Zhelyazkov \\ Faculty of Physics, Sofia University, BG-1126 Sofia, Bulgaria \\ Z. Naturforsch. 42 a, $1443-1450$ (1987); received July 24, 1986
}

\begin{abstract}
We study the propagation of surface/pseudosurface modes in the structured solar atmosphere assuming that the surface waves may be able to heat the chromosphere and corona. The wave energy can be dissipated by radiation, ion viscosity, and electron heat conduction. For the solar corona, it is found that the pseudosurface waves, trapped in the coronal loops, dissipate efficiently only if their periods are longer than 200 seconds and only if the background magnetic field is smaller than 5 gauss.
\end{abstract}

\section{Introduction}

The mechanisms which convert the kinetic energy of the solar photosphere and convection zone into the thermal energy of the chromosphere and corona have been under study for a long time [1]. Coronal heating by MHD waves has been widely investigated. The MHD slow modes (Alfvén and slow magnetosonic waves), however, can probably be ignored in this context, since they propagate too slowly to carry the required energy flux into the corona, subject to the constraints imposed by the observed amplitudes of nonthermal motions in the corona and underlying chromosphere. The fast magnetosonic wave has a number of appealing features. Its group velocity is large, and it is therefore in principle capable of carrying a large energy flux in the corona. It is intrinsically compressive and thus subject to dissipation by viscosity, heat conduction, and radiation [2]. Recent studies of Leroy and Schwartz $[3,4]$ on propagation of bulk fast MHD waves (particularly magneto-acoustic gravity oscillations) in a model of the solar atmosphere which includes the chromosphere, the transition region and the corona show that these fast modes cannot supply the required energy flux into the corona.

A remaining possibility takes advantage of observations that the corona is highly structured. The magnetic atmosphere of the solar corona can be viewed as made up of coronal loops (magnetic flux tubes), the transverse dimensions of which are much shorter than

Reprint requests to Prof. Dr. I. Zhelyazkov, Faculty of Physics, 5 Anton Ivanov Blvd., BG-1126 Sofia, Bulgaria.

* Permanent address: Department of Physics, Faculty of Science, AL-BAATH University, Homs, Syria. longitudinal ones. The atmosphere is dominated by magnetic forces; the flow of heat is principally along the field lines. Density inhomogeneities also occur. As a result, a magnetic flux tube can become visible, standing out from its neighbours [5], even though the whole of the atmosphere is permeated by a magnetic field.

Inhomogeneities in the magnetic field induction are probably not large, but even in a uniform field strong density variations will result in strong differences in Alfvén speed, and it is the Alfvén speed, rather than the field itself, that governs the wave propagation. In a structured atmosphere, where discontinuities of densities, velocities etc. exist, new aspects immediately arise; in particular, the existence of discontinuities allows the propagation of surface modes. These modes have properties which may make them capable of heating the chromosphere and corona.

In this paper, we evaluate the extent to which fast magnetosonic surface waves can be collisionally dissipated in the chromosphere and corona, i.e. we calculate their dissipation by viscosity, heat conduction, and radiation. It is interesting to note that viscosity can contribute a much more important dissipative term than electrical resistivity [6]. Since we are considering collisional dissipation, we will confine our attention to dense chromospheric/coronal regions, where collisions are frequent; coronal holes will be ecxluded from our analysis.

\section{Basic Assumptions, Governing Equations, and Surface-Wave Dispersion Relation}

The dissipation of magnetoacoustic surface waves in a magnetic cylinder embedded in a magnetic envi-

0932-0784 / 87 / 1200-1443\$01.30/0. - Please order a reprint rather than making your own copy. 
ronment is analyzed subject to a number of simplifying assumptions:

(i) The effect of gravity will be ignored, our emphasis being on an exploration of the role of magnetic/ density structuring. We will concentrate our attention on the two cases that most closely model solar features, i. e. isolated flux tubes of chromospheric fibrils and coronal magnetic loops. For these the neglect of gravity and, associated with it, stratification might be reasonable.

(ii) The magnetic field pressure in the chromosphere and corona is assumed to dominate the thermal pressure. Nevertheless, we will assume that the propagation of adiabatic waves is governed by the usual equations of ideal MHD.

(iii) The surface waves are assumed to be weakly dissipative, i.e. the imaginary part of the wavenumber is much smaller than the real part of the wavevector component along the background magnetic field, $\boldsymbol{B}_{0} \equiv B_{0} \hat{z}$. This permits an approximate procedure [2] for calculating the so called dissipation distance $\equiv$ (imaginary part of the wavenumber) $)^{-1}$. The wave properties are first calculated in the absence of dissipation. Then, given those wave properties, it is possible to calculate the rate at which the waves lose energy to radiation and to heat via viscosity and heat conduction. Finally, the dissipation length is determined by equating the volumetric energy loss rate to the divergence of the surface wave Poynting flux. This procedure obviates the need to calculate a full wave dispersion relationship in the presence of viscosity, heat conduction and radiation, but it is limited to the weak damping limit.

(iv) The plasma density is assume to possess discontinuities only in radial direction; both density and background magnetic field are taken to be uniform in the $z$-direction.

(v) The background flow velocity is assumed to be zero.

We represent a chromospheric fibril or a coronal loop by a straight vertical magnetic cylinder of radius $a$, field induction $B_{0}$, gas density $\varrho_{0}$, gas pressure $p_{0}$, and temperature $T_{0}$. Outside the cylinder, the magnetic field is $B_{\mathrm{e}} \approx B_{0}$, the gas density $\varrho_{\mathrm{e}} \ll \varrho_{0}$, the pressure $p_{\mathrm{e}}$, and the temperature $T_{\mathrm{e}}$. In deriving the wave dispersion relation we will follow Edwin and Roberts [7]. For clarity and completeness, some of our expressions for different wave and media quantities will overlap those of Edwin and Roberts, but we stress on some features as well.
In the absence of dissipation, the linearized MHD equations are:

$\frac{\partial \varrho}{\partial t}+\nabla \cdot\left(\varrho_{0} v\right)=0$,

$\varrho_{0} \frac{\partial \boldsymbol{v}}{\partial t}=-\nabla\left(p+\frac{1}{4 \pi} \boldsymbol{B}_{0} \cdot \boldsymbol{b}\right)+\frac{1}{4 \pi}\left(\boldsymbol{B}_{0} \cdot \nabla\right) \boldsymbol{b}$,

$\frac{\partial \boldsymbol{b}}{\partial t}=\nabla \times\left(\boldsymbol{v} \times \boldsymbol{B}_{0}\right)$,

$\frac{\partial p}{\partial t}+\boldsymbol{v} \cdot \nabla p_{0}=c_{0}^{2}\left(\frac{\partial \varrho}{\partial t}+v \cdot \nabla p_{0}\right)$,

where $\boldsymbol{b}$ is the perturbed (wave) magnetic field, $c_{0}=\left(\gamma p_{0} / \varrho_{0}\right)^{1 / 2}$ the sound speed (inside the cylinder) and $\gamma$ the ratio of the specific heats. The other notation is standard. With the assumption $p_{0}=$ const, (4) becomes

$$
\frac{\partial p}{\partial t}=-\varrho_{0} c_{0}^{2} \Delta,
$$

where $\Delta \equiv \nabla \cdot \boldsymbol{v}$. On the other hand the pressure balance implies

$$
p_{0}+\frac{B_{0}^{2}}{8 \pi}=p_{\mathrm{e}}+\frac{B_{\mathrm{e}}^{2}}{8 \pi}
$$

and the perturbed total pressure is

$$
p_{\text {total }}=p+\frac{B_{0}}{4 \pi} b_{z} .
$$

The set of (1) - (4) can be reduced to the single equation

$$
\begin{aligned}
& \frac{\partial^{2}}{\partial t^{2}}\left\{\left[\frac{\partial^{2}}{\partial t^{2}}-\left(c_{0}^{2}+v_{\mathrm{A}}^{2}\right) \nabla^{2}\right] \Delta\right\} \\
& +c_{0}{ }^{2} v_{\mathrm{A}}^{2} \frac{\partial^{2}}{\partial z^{2}} \nabla^{2} \Delta=0,
\end{aligned}
$$

where $v_{\mathrm{A}}=B_{0} /\left(4 \pi \varrho_{0}\right)^{1 / 2}$ is the Alfvén speed inside the cylinder.

If the solution of (6) is sought in the form

$$
\Delta=R(r) \exp (-i \omega t+i n \theta+i k z)
$$

then (6) implies that $R(r)$ satisfies the Bessel equation

$$
\frac{1}{r} \frac{\partial}{\partial r} r \frac{\partial R}{\partial r}+\left(n_{0}^{2}-\frac{n^{2}}{r^{2}}\right) R=0,
$$

where

$$
n_{0}^{2}=\frac{k^{2} c_{0}^{2}-\omega^{2}}{c_{0}^{2}+v_{\mathrm{A}}^{2}} \frac{k^{2} v_{\mathrm{A}}^{2}-\omega^{2}}{\omega^{2}-k^{2} c_{\mathrm{T}}^{2}}
$$


with

$$
c_{\mathrm{T}}=\frac{c_{0} v_{\mathrm{A}}}{\left(c_{0}^{2}+v_{\mathrm{A}}^{2}\right)^{1 / 2}}
$$

being the so called tube or cusp speed [7] and

$$
R(r)=A_{0} J_{n}\left(n_{0} r\right), \quad n_{0}^{2}>0, \quad r<a .
$$

Here $A_{0}$ is a constant and $J_{n}$ is the Bessel function of order $n$.

In the external region, supposing that there is no propagation of energy away from (or towards) the cylinder, $r=a$, the behaviour of $R(r)$ is governed by the equation

$$
\frac{1}{r} \frac{\partial}{\partial r} r \frac{\partial R}{\partial r}-\left(m_{\mathrm{e}}^{2}+\frac{n^{2}}{r^{2}}\right) R=0
$$

and we take

$$
R(r)=A_{\mathrm{e}} K_{n}\left(m_{\mathrm{e}} r\right), \quad m_{\mathrm{e}}^{2}>0, \quad r>a,
$$

where $A_{\mathrm{e}}$ is a constant, $K_{n}$ is the modified Bessel function of second kind and

$$
m_{\mathrm{e}}^{2}=\frac{k^{2} c_{\mathrm{e}}^{2}-\omega^{2}}{c_{\mathrm{e}}^{2}+v_{\mathrm{Ae}}^{2}} \frac{\omega^{2}-k^{2} v_{\mathrm{Ae}}^{2}}{\omega^{2}-k^{2} c_{\mathrm{Te}}^{2}}
$$

with

$$
c_{\mathrm{Te}}=\frac{c_{\mathrm{e}} v_{\mathrm{Ae}}}{\left(c_{\mathrm{e}}^{2}+v_{\mathrm{Ae}}^{2}\right)^{1 / 2}} .
$$

Here $c_{\mathrm{e}}=\left(\gamma p_{\mathrm{e}} / \varrho_{\mathrm{e}}\right)^{1 / 2}$ and $v_{\mathrm{Ae}}=B_{\mathrm{e}} /\left(4 \pi \varrho_{\mathrm{e}}\right)^{1 / 2}$ are the sound and Alfvén speeds outside the cylinder.

Continuity of the radial velocity component

$$
v_{\mathrm{r}}=\frac{c_{0}^{2}+v_{\mathrm{A}}^{2}}{k^{2} v_{\mathrm{A}}^{2}-\omega^{2}} \frac{\partial}{\partial r} \Delta
$$

and the total pressure

$$
p_{\text {total }}=\frac{\varrho}{i \omega}\left(c_{0}^{2}+v_{\mathrm{A}}^{2}\right) \Delta
$$

across the interface $r=a$ then yields the required wave dispersion relationship:

$$
\begin{aligned}
& \varrho_{0}\left(k^{2} v_{\mathrm{A}}^{2}-\omega^{2}\right) m_{\mathrm{e}} \frac{K_{n}^{\prime}\left(m_{\mathrm{e}} a\right)}{K_{n}\left(m_{\mathrm{e}} a\right)} \\
& =\varrho_{\mathrm{e}}\left(\mathrm{k}^{2} v_{\mathrm{Ae}}^{2}-\omega^{2}\right) n_{0} \frac{J_{n}^{\prime}\left(n_{o} a\right)}{J_{n}\left(n_{0} a\right)} .
\end{aligned}
$$

The dash denotes the derivative of a Bessel function with respect to its argument. We note that in deriving the expression for $v_{\mathrm{r}}$ we took $\partial v_{z} / \partial z=0$, which is rea- sonable for the problem at hand (i.e. at $c_{0}^{2} \ll v_{\mathrm{A}}^{2}$ ). The dispersion relation (13) governs the propagation of waves whose fields are oscillatory inside the cylinder and decaying outside. Edwin and Roberts call them body waves [7], but they should be termed pseudosurface waves [8].

Further we will confine our attention to the so called kink modes corresponding to the value $n=1$. According to [7] a magnetic cylinder has two kink pseudosurface modes, a fast and a slow mode. Under coronal conditions, $B_{\mathrm{e}} \cong B_{0}, p_{0}=p_{\mathrm{e}}$, and $\varrho_{0} \gg \varrho_{\mathrm{e}}$, the fast kink mode has a phase speed close to

$$
c_{k}=\left(\frac{\varrho_{0} v_{\mathrm{A}}^{2}+\varrho_{\mathrm{e}} v_{\mathrm{Ae}}^{2}}{\varrho_{0}+\varrho_{\mathrm{e}}}\right)^{1 / 2} .
$$

Since we are interested in wave propagation on coronal loops, (14) suggests that dense loops in the corona are able to sustain free oscillations with characteristic periods on an Alfvénic time-scale (as $v_{\mathrm{A}}<\omega / k<v_{\mathrm{Ae}}$ ). It turns out that the same situation is valid for the chromospheric fibrils. A simple expression for the wave dispersion relation can be derived from (13) in the limit of a thin cylinder, $|k| a \ll 1$, and it is [7]:

$$
\begin{aligned}
\frac{\omega}{k} \cong c_{k}\left\{1-\frac{1}{2} \frac{\varrho_{0} \varrho_{\mathrm{e}}}{\left(\varrho_{0}+\varrho_{\mathrm{e}}\right)^{2}} \frac{v_{\mathrm{Ae}}^{2}-v_{\mathrm{A}}^{2}}{c_{\mathrm{k}}^{2}}\right. \\
\left.\quad \times \lambda_{\mathrm{e}}^{2}(k a)^{2} K_{0}\left(\lambda_{\mathrm{e}}|\mathrm{k}| a\right)\right\},
\end{aligned}
$$

where

$$
\lambda_{\mathrm{e}}^{2} \equiv \frac{m_{\mathrm{e}}^{2}}{k^{2}}=\frac{c_{\mathrm{e}}^{2}-c_{k}^{2}}{c_{\mathrm{e}}^{2}+v_{\mathrm{Ae}}^{2}} \cdot \frac{v_{\mathrm{Ae}}^{2}-c_{k}^{2}}{c_{\mathrm{Te}}^{2}-c_{k}^{2}} .
$$

We note that the fast-kink phase velocity is almost constant and this is illustrated on Fig. 4 of [7]. Both for coronal and chromospheric conditions $\left(B_{0} \cong B_{\mathrm{e}}\right)$ the dispersion relation (15) can be simplified to the form

$\frac{\omega}{k} \cong c_{k}\left[1-\frac{1}{4} \frac{\varrho_{0}-\varrho_{\mathrm{e}}}{\varrho_{0}+\varrho_{\mathrm{e}}} \lambda_{\mathrm{e}}^{2}(k a)^{2} K_{0}\left(\lambda_{\mathrm{e}}|k| a\right)\right]$,

where

$$
c_{k}=\frac{B_{0}}{\left[2 \pi\left(\varrho_{0}+\varrho_{\mathrm{e}}\right)\right]^{1 / 2}}
$$

is obviously a mean Alfvén speed for the inhomogeneous medium. We end this section with the comment that the fast kink pseudosurface waves (13) in a low- $\beta$ plasma are cylindrical equivalents of Love waves [9] in seismology [5]. 


\section{Dissipation Length of Fast Kink Surface Mode}

As discussed in Sect. II, we wish to calculate the dissipation of fast magnetoacoustic surface wave by viscosity, heat conduction, and radiation. [10]

$$
Q_{\text {vis }}=\frac{\eta_{0}}{3} \Delta^{2}
$$

where we make use of fact that $\partial v_{z} / \partial z=0$ and that the ions undergo many cyclotron orbits between collisions. The viscosity is mainly due to the protons, and

$$
\eta_{0}=1.0 \times 10^{-16} T_{0}^{5 / 2}
$$

(in cgs units, $T_{0}$ in Kelvins) is the most important of the five viscosity coefficients given by Braginskii [10] (for a Coulomb logarithm of 22).

The heat conduction flux is carried mainly by the electrons. If the electrons are magnetized, the volumetric heating rate due to heat conduction, when the wave frequency is relatively small (and the waves induce nearly adiabatic compressions in the plasma), is [2]

$$
Q_{\text {ther }}=x_{\| \mathbf{e}}\left(\frac{k}{\omega}\right)^{2} T_{0}(\gamma-1)^{2} \Delta^{2},
$$

where [10]

$$
\varkappa_{\| \mathrm{e}}=8.4 \times 10^{-7} T_{0}^{5 / 2} ;
$$

the coulomb logarithm is again taken to be 22 . The value of $\gamma$ we shall assume to be $5 / 3$.
We consider that the plasma is optically thin and radiates $\Lambda N_{0}^{2} \mathrm{erg} \mathrm{cm}^{-3} \mathrm{~s}^{-1}$, where $N_{0}$ is the electron number density (in $\mathrm{cm}^{-3}$ ) and $\Lambda$ is a temperaturedependent factor which we take to be $10^{-24}$ $\mathrm{erg} \mathrm{cm} \mathrm{cm}^{3}$ at photospheric temperatures and $10^{-22}$ $\mathrm{erg} \mathrm{cm} \mathrm{s}^{3} \mathrm{~s}^{-1}$ at chromospheric and coronal ones [11]. The volumetric rate at which radiation extracts energy from the waves in given by [12]

$$
Q_{\mathrm{rad}}=\Lambda N_{0}^{2} \omega^{-2} \Delta^{2} \text {. }
$$

Equations (16)-(18) are the desired expressions for the volumetric rates at which the waves lose energy to the plasma and radiation field.

The next step in the calculation requires knowledge of the Poynting flux carried by the surface wave. Since the magnetosonic wave has both a longitudinal (acoustic) and transverse mode [13], the time averaged $z$-component of the Poynting flux is

$$
\begin{aligned}
\left\langle S_{\mathrm{z}}\right\rangle= & \frac{1}{2} \operatorname{Re} \int_{0}^{\infty} \mathrm{d} r r \int_{0}^{2 \pi} \mathrm{d} \theta p_{\text {total }} \boldsymbol{v}^{*} \cdot \hat{z} \\
& +\frac{c}{8 \pi} \operatorname{Re} \int_{0}^{\infty} \mathrm{d} r r \int_{0}^{2 \pi} \mathrm{d} \theta\left(\boldsymbol{E}^{*} \times \boldsymbol{b}\right) \cdot \hat{z} .
\end{aligned}
$$

If the wave electric field is given by

$$
\boldsymbol{E}^{*}=-\frac{1}{c} \boldsymbol{v}^{*} \times \boldsymbol{B}_{0}
$$

after some lengthly but straightforward algebra we derive

$$
\begin{aligned}
&\left\langle\bar{S}_{z}\right\rangle= \frac{\pi}{2} \frac{1}{c_{k} k^{2}}\left\{\varrho_{0} c_{0}^{2} A_{0}^{2} D_{0} \int_{0}^{a} \mathrm{~d} r r J_{1}^{2}\left(n_{0} r\right)-\varrho_{\mathrm{e}} c_{\mathrm{e}}^{2} A_{\mathrm{e}}^{2} D_{\mathrm{e}} \int_{a}^{\infty} \mathrm{d} r r K_{1}^{2}\left(m_{\mathrm{e}} r\right)\right\} \\
&+\frac{1}{8} \frac{B_{0}^{2}}{c_{k} k^{4}}\left\{n_{0}^{2} A_{0}^{2} D_{0}^{2} \int_{0}^{a} \mathrm{~d} r r J_{1}^{\prime 2}\left(n_{0} r\right)+m_{\mathrm{e}}^{2} A_{\mathrm{e}}^{2} D_{\mathrm{e}}^{2} \int_{a}^{\infty} \mathrm{d} r r K_{1}^{\prime 2}\left(m_{\mathrm{e}} r\right)+A_{0}^{2} D_{0}^{2} \int_{0}^{a} \mathrm{~d} r \frac{J_{1}^{2}\left(n_{0} r\right)}{r}\right. \\
&\left.+A_{\mathrm{e}}^{2} D_{\mathrm{e}}^{2} \int_{a}^{\infty} \mathrm{d} r \frac{K_{1}^{2}\left(m_{\mathrm{e}} r\right)}{r}\right\},
\end{aligned}
$$

where

$$
D_{0}=\frac{c_{0}^{2}+v_{\mathrm{A}}^{2}}{c_{k}^{2}-v_{\mathrm{A}}^{2}} \quad \text { and } \quad D_{\mathrm{e}}=\frac{c_{\mathrm{e}}^{2}+v_{\mathrm{Ae}}^{2}}{v_{\mathrm{Ae}}^{2}-c_{k}^{2}}
$$

and the overbar denotes the average over the azimuthal coordinate $(n=1)$. The final form $\left\langle\bar{S}_{z}\right\rangle$, by setting $A_{0}=\alpha A_{\mathrm{e}}$ with

$$
\alpha \equiv \frac{\varrho_{\mathrm{e}}}{\varrho_{0}} \frac{c_{\mathrm{e}}^{2}+v_{\mathrm{Ae}}^{2}}{c_{0}^{2}+v_{\mathrm{A}}^{2}} \frac{K_{1}\left(m_{\mathrm{e}} a\right)}{J_{1}\left(n_{0} a\right)},
$$


is

$$
\begin{aligned}
\left\langle\bar{S}_{z}\right\rangle=\frac{\pi \varrho_{0} A_{\mathrm{e}}^{2}}{4 c_{k} k^{4}}\left[( k a ) ^ { 2 } \left\{c_{0}^{2} \alpha^{2} D_{0}\left[J_{0}^{2}\left(n_{0} a\right)+J_{1}^{2}\left(n_{0} a\right)-\frac{2}{n_{0} a} J_{0}\left(n_{0} a\right) J_{1}\left(\mathrm{n}_{0} a\right)\right]\right.\right. \\
\left.\quad-\frac{\varrho_{\mathrm{e}}}{\varrho_{0}} c_{\mathrm{e}}^{2} D_{\mathrm{e}}\left[K_{0}^{2}\left(m_{\mathrm{e}} a\right)-K_{1}^{2}\left(m_{\mathrm{e}} a\right)+\frac{2}{m_{\mathrm{e}} a} K_{0}\left(m_{\mathrm{e}} a\right) K_{1}\left(m_{\mathrm{e}} a\right)\right]\right\} \\
+v_{\mathrm{A}}^{2}\left\{\alpha^{2} D_{0}^{2}\left[n_{0}^{2} a^{2} J_{0}^{2}\left(n_{0} a\right)-\left(2-n_{0}^{2} a^{2}\right) J_{1}^{2}\left(n_{0} a\right)\right]\right. \\
\left.\left.+D_{\mathrm{e}}^{2}\left[\left(2+m_{\mathrm{e}}^{2} a^{2}\right) K_{1}^{2}\left(m_{\mathrm{e}} a\right)-m_{\mathrm{e}}^{2} a^{2} K_{0}^{2}\left(m_{\mathrm{e}} a\right)\right]\right\}\right] .
\end{aligned}
$$

The damping lenght of the waves is calculated by equating the divergence of the Poynting flux to the negative of the volumetric heating and radiation rates, i.e.

$$
\frac{\mathrm{d}\left\langle\bar{S}_{z}\right\rangle}{\mathrm{d} z}=-\langle\bar{Q}\rangle,
$$

where

$$
\begin{aligned}
&\langle\bar{Q}\rangle=\left\langle\bar{Q}_{\mathrm{vis}}\right\rangle+\left\langle\bar{Q}_{\mathrm{ther}}\right\rangle+\left\langle\bar{Q}_{\mathrm{rad}}\right\rangle \\
&=\frac{\pi a^{2} A_{\mathrm{e}}^{2}}{4}\left\{\alpha^{2}\left[J_{0}^{2}\left(n_{0} a\right)+J_{1}^{2}\left(n_{0} a\right)-\frac{2}{n_{0} a} J_{0}\left(n_{0} a\right) J_{1}\left(n_{0} a\right)\right]+K_{0}^{2}\left(m_{\mathrm{e}} a\right)-K_{1}^{2}\left(m_{\mathrm{e}} a\right)\right. \\
&\left.\quad+\frac{2}{m_{\mathrm{e}} a} K_{0}\left(m_{\mathrm{e}} a\right) K_{1}\left(m_{\mathrm{e}} a\right)\right\}\left(\frac{\eta_{0}}{3}+x_{\| \mathrm{e}}\left(\frac{k}{\omega}\right)^{2} T_{0}(\gamma-1)^{2}+\Lambda\left(\frac{N_{0}}{\omega}\right)^{2}\right)
\end{aligned}
$$

is the dissipated power per unit cylinder length. Thus, if we denote the e-folding distance for the Poynting flux by $L_{z}$, we have

$$
L_{z}=\left\langle\bar{S}_{z}\right\rangle /\langle\bar{Q}\rangle \text {. }
$$

Taking the corresponding expressions for $\left\langle\bar{S}_{z}\right\rangle$ and $\langle\bar{Q}\rangle$, from (22) we get

$$
\begin{aligned}
L_{z}=\frac{\varrho_{0}}{c_{k}(k a)^{2} k^{2}} \mathbb{E}(k a)^{2}\left\{c_{0}^{2} \alpha^{2} D_{0}\left[J_{0}^{2}\left(n_{0} a\right)+J_{1}^{2}\left(n_{0} a\right)-\frac{2}{n_{0} a} J_{0}\left(n_{0} a\right) J_{1}\left(n_{0} a\right)\right]\right. \\
\left.\quad-\frac{\varrho_{\mathrm{e}}}{\varrho_{0}} c_{\mathrm{e}}^{2} D_{\mathrm{e}}\left[K_{0}^{2}\left(m_{\mathrm{e}} a\right)-K_{1}^{2}\left(m_{\mathrm{e}} \mathrm{a}\right)+\frac{2}{m_{\mathrm{e}} a} K_{0}\left(m_{\mathrm{e}} a\right) K_{1}\left(m_{\mathrm{e}} a\right)\right]\right\} \\
\left.+v_{\mathrm{A}}^{2}\left\{\alpha^{2} D_{0}^{2}\left[n_{0}^{2} a^{2} J_{0}^{2}\left(n_{0} a\right)-\left(2-n_{0}^{2} a^{2}\right) J_{1}^{2}\left(n_{0} a\right)\right]+D_{\mathrm{e}}^{2}\left[\left(2+m_{\mathrm{e}}^{2} a^{2}\right) K_{1}^{2}\left(m_{\mathrm{e}} a\right)-m_{\mathrm{e}}^{2} a^{2} K_{0}^{2}\left(m_{\mathrm{e}} a\right)\right]\right\}\right] \\
\times\left\{\alpha^{2}\left[J_{0}^{2}\left(n_{0} a\right)+J_{1}^{2}\left(n_{0} a\right)-\frac{2}{n_{0} a} J_{0}\left(n_{0} a\right) J_{1}\left(n_{0} a\right)\right]+K_{0}^{2}\left(m_{\mathrm{e}} a\right)-K_{1}^{2}\left(m_{\mathrm{e}} a\right)\right.
\end{aligned}
$$

where the value of $\omega / k$ has been supposed to be equal to $c_{k}$. This formula shows that the magnitude of $L_{z}$ depends on the magnetic field $B_{0}$ (via $\left.v_{\mathrm{A}}\right)$ in the sence that the waves dissipate much less efficiently in regions of strong magnetic field, and vice-versa. The ability of surface modes to damp collisonally and heat the chromosphere or corona will therefore be limited to weak magnetic fields, as for example in quiet coronal regions. 
Formula (22 a) applies both to chromospheric and coronal conditions. Giovanelli [14] has described in some observational detail the propagation of waves in chromospheric fibrils with periods of about $2 \pi / \omega=170 \mathrm{~s}$ and speeds of roughly $70 \mathrm{~km} \mathrm{~s}^{-1}$. Giovanelli pointed out that the disturbances, though transverse to the fibrils, involve intensity fluctuations and so are not Alfvén waves. Furthermore, the wavefronts appear to cover only the width of a single fibril. Wentzel [15] has suggested that fibril waves are likely to be surface modes propagating along a magnetic tube, the tube having an internal Alfven speed that is lower than the surroundings.

For a low- $\beta$ plasma (in chromosphere and corona) one would regard fibril waves as an example of a fast pseudosurface kink mode or as a propagating magnetic Love wave [7]. Typical values of the basic plasma parameters in the chromosphere (at a height of $2100 \mathrm{~km})$ are [16]: $T=22000 \mathrm{~K}\left(T_{\mathrm{e}} \approx T_{0}\right), N_{\mathrm{e}}=$ $2.896 \times 10^{10} \mathrm{~cm}^{-3}, p_{\mathrm{e}}=0.184$ dyne $\mathrm{cm}^{-2}, \varrho_{\mathrm{e}}=6.718$ $\times 10^{-14} \mathrm{~g} \mathrm{~cm}^{-3}$. (These parameters correspond to Model D of [16], and they are close to those of Sobolev [17], although the electron number density $N_{\mathrm{e}}$ given in [17] seems to be too high.) Assuming $\varrho_{0} / \varrho_{\mathrm{e}}=4$ (i. e. $\varrho_{0}=2.687 \times 10^{-13} \mathrm{~g} \mathrm{~cm}^{-3}$ and $N_{0}=1.16 \times 10^{11}$ $\mathrm{cm}^{-3}$ ), a phase velocity of the fast kink mode of order $70 \mathrm{~km} \mathrm{~s}^{-1}$ might be obtained from expression (14a) at $B_{0} \approx B_{\mathrm{e}}=100 \mathrm{G}\left(c_{k}=6.88 \times 10^{6} \mathrm{~cm} \mathrm{~s}^{-1}\right)$. Further, taking the tube radius $a=700 \mathrm{~km}$ and the wavenumber $k=\omega / c_{k}=5.37 \times 10^{-9} \mathrm{~cm}^{-1} \quad(k a=0.376 \ll 1)$, from ( $22 \mathrm{a}$ ) we have $L_{z}=1.44 \times 10^{9} \mathrm{~cm}$. We note that: (i) under chromospheric conditions the radiation losses are much larger than the dissipation due to the viscosity and heat conduction; (ii) the dissipation length $L_{z}$ proves to be longer than the length of fibrils $\sim 7000 \mathrm{~km}$.

The fast magnetosonic waves arise in the corona as a free mode (with real frequency and wavenumber) only for a loop with an Alfvén speed that is lower than in its surroundings. Thus, actually, only high density loops may act as wave ducts trapping fast pseudosurface waves. According to Roberts, Edwin, and Benz [5] one can suppose that the waves occur as standing modes in a loop of length $L$, the foot-points of which are firmly anchored in the high-density chromospheric atmosphere (we remember that the effects of loop curvature and gravitational stratification are ignored in order to exhibit clearly the important role of field/density structuring of magnetic oscillations). With disturbances assumed zero at the ends $(z=0, L)$ of the loop, one may take $k=j \pi / L$ for integers $j=1,2,3, \ldots$ The period $\tau=2 \pi / \omega$ of the fast magnetosonic kink mode is given by

$$
\begin{aligned}
\tau & =\frac{2 L}{j c_{k}}=\frac{2 \sqrt{2 \pi}}{j} \frac{L}{B_{0}}\left(\varrho_{0}+\varrho_{\mathrm{e}}\right)^{1 / 2} \\
& =\frac{\left(8 \pi \varrho_{0}\right)^{1 / 2}}{j} \frac{L}{B_{0}}\left(1+\frac{\varrho_{\mathrm{e}}}{\varrho_{0}}\right)^{1 / 2} .
\end{aligned}
$$

The integer $j$, which determines the number of nodes in the oscillations along the loop, may be taken, say, 1 or 2 ; these being the modes most easily to excite. Taking $\varrho_{\mathrm{e}} / \varrho_{0}=1 / 4$, with the help of the relation $\varrho_{0}=1.944 \mu m_{\mathrm{H}} N_{0} \quad$ (where $\quad \mu=0.5724 \quad$ and $\left.m_{\mathrm{H}}=1.66 \times 10^{-24} \mathrm{~g}\right)(23)$ becomes

$$
\tau=\frac{7.62 \times 10^{-12} L N_{0}^{1 / 2}}{j B_{0}} .
$$

We will use (23a) in order to estimate the magnitude of the magnetic field $B_{0}$ required for sustaining oscillations (standing waves) of period $\tau$. (The numerical factor in (23a) in front of $10^{-12}$ differs slightly from that in expression (5) in [5]; this difference is due to the fact that Roberts, Edwin, and Benz have assumed $\varrho_{\mathrm{e}} / \varrho_{0} \rightarrow 0$ and have used a simpler relation between $\varrho_{0}$ and $N_{0}$.)

We consider some observational studies of coronal oscillations that may be related to the fast kink surface waves. Recently Koutchmy, Žugžda, and Ločans [18] reported periodicities of $43 \mathrm{~s}, 80 \mathrm{~s}$ and $300 \mathrm{~s}$ in power spectra obtained in the green coronal line $5303 \AA$ of Fe XIV. They evaluate $N_{0}=2.5 \times 10^{8} \mathrm{~cm}^{-3}$ and $T_{0}=1.8 \times 10^{6} \mathrm{~K}$. Dermendjiev et al. [19], analyzing the periodicities of the same coronal line on the basis of a series of 86 photographs, have concluded that there exist 280- and 360-second oscillations (shorter periods of $120 \mathrm{~s}$ and $60 \mathrm{~s}$ can also be observed, but they are not so noticeable). The same authors give [20] the following value for the temperature in the solar corona: $T_{0}=2.43 \times 10^{6} \mathrm{~K}$. In a recent comment on the solar atmosphere parameters Athay [21] states that the gas pressure in the corona should be $\sim 0.1$ dyne $\mathrm{cm}^{-2}$ and the temperature $T_{0}=2 \times 10^{6} \mathrm{~K}$, and in accordance with him we will take $\mathrm{N}_{0}=$ $1.8 \times 10^{8} \mathrm{~cm}^{-3}$. Assuming $j=2$ and $L=1.6 \times 10^{10} \mathrm{~cm}$, (23a) yields $B_{0}=19 \mathrm{G}, 10 \mathrm{G}$ and $\sim 3 \mathrm{G}$ for the above mentioned values of $\tau$ obtained in [18]. The magnetic field of $3 \mathrm{G}$, required for the occurence of standing waves of period $\tau=300 \mathrm{~s}$, is close to those magnetic 
fields which exist in the quiet corona [21]. So, after calculating all speeds for 300 -second oscillations $\left(c_{k}=5.87 \times 10^{7} \mathrm{~cm} \mathrm{~s}^{-1}\right.$ for example) and taking $a=1.6 \times 10^{9} \mathrm{~cm}$, from (22a) at $k=3.6 \times 10^{-10} \mathrm{~cm}^{-1}$ $(k a=0.576<1)$ we get $L_{z}=1.40 \times 10^{12} \mathrm{~cm}$. This distance is two orders larger than the loop length $L$, which means that at standing wave pattern a noticeable decrease of the wave amplitude occurs after several consecutive reflections; this is consistent with the assumption that the waves are weakly damped. The shorter-period-pseudosurface modes should exist at stronger magnetic fields, and the waves have very long dissipation lengths; such waves are then not suitable for coronal heating.

\section{Discussion and Conclusion}

It is now accepted that the sun probably uses its enormous magnetic energy content for heating the corona. Such an energy conversion can take place both by magnetic reconnection in current sheets [22] and also by the dissipation of MHD oscillations, which naturally fill the whole atmosphere and which can transport energy up from low atmospheric levels. Our calculations have shown that the structured solar atmosphere can support the propagation of surface or pseudosurface waves which might be dissipated by the medium. The crude model of fibrils as vertical thin cylinders being denser than the outer region in the chromosphere confirms the suggestion that they act as waveguides. The wave dissipation is due mainly to radiative damping $\left(\left\langle\bar{Q}_{\mathrm{rad}}\right\rangle\right.$ is so large compared to $\left\langle\bar{Q}_{\text {vis }}\right\rangle$ and $\left\langle\bar{Q}_{\text {ther }}\right\rangle$ that the the latter mechanisms might be neglected in (21)), which is in agreement with the conclusions of Cally [23], made on the basis of a more realistic model (waves traveling along a horizontal magnetic field permeated by gravity).

In coronal conditions the energy of fast magnetosonic pseudosurface waves (kink mode) is transfered to the medium through the ion viscosity and heat conduction. Braginskii's [10] equation (16) for the viscous heating rate is valid for a collisional plasma which requires

$$
\omega \tau_{\mathrm{p}}<1,
$$

where $\tau_{\mathrm{p}}$ is the proton collision time. In our case (hydrogen plasma with a Coulomb logarithm of 22) we have [10]

$$
\tau_{\mathrm{p}}=0.75 T_{0}^{3 / 2} N_{0}^{-1} \mathrm{~s} .
$$

At $T_{0}=2 \times 10^{6} \mathrm{~K}$ and $N_{0}=1.8 \times 10^{8} \mathrm{~cm}^{-3}$ the corresponding value of $\tau_{\mathrm{p}}$ is $11.8 \mathrm{~s}$. Since for the typical five-minute oscillations $\omega \cong 0.021 \mathrm{~s}^{-1}$, (24) is fulfilled $\left(\omega \tau_{\mathrm{p}}=0.25\right)$. We notice that the viscosity can be effective as a mechanism for energy wave dissipation in the corona only for long-period oscillations $(\omega / 2 \pi>$ $150 \div 200$ s).

Similarly, (17) for the heating due to heat conduction is valid only if the electron mean free path, $\lambda_{\mathrm{e}}$, is short enough so that

$$
k \lambda_{\mathrm{e}}<1 .
$$

Bearing in mind that $[10]$

$$
\lambda_{\mathrm{e}}=4.9 \times 10^{3} T_{0}^{2} N_{0}^{-1} \mathrm{~cm},
$$

i. e. for the problem at hand $\lambda_{\mathrm{e}} \cong 1.1 \times 10^{8} \mathrm{~cm}$, with $k=3.6 \times 10^{-10} \mathrm{~cm}^{-1}$ we have $k \lambda_{\mathrm{e}}=0.04$. This means that at typical coronal conditions and at lower frequencies the heat conduction dominates the viscosity. The latter is confirmed by comparing $\left\langle\bar{Q}_{\text {vis }}\right\rangle$ and $\left\langle\bar{Q}_{\text {ther }}\right\rangle$ in calculating $L_{z}$. In contrast to the chromospheric fibrils, the wave damping in coronal loops due to radiation is small.

As we have mentioned, the fast magnetosonic waves, due to their hybrid nature, carry both acoustic and electromagnetic energy flux. For chromospheric and coronal conditions, however, the contribution of the acoustic part to the wave power, $\left\langle\bar{S}_{z}\right\rangle$, is negligibly small. Thus, for a rough estimation of the dissipation length, $L_{z}$, one can drop in (22a) the terms (in the brackets) multiplied by $c_{0}^{2}$ and $c_{\mathrm{e}}^{2}$.

A "weak" point of the mechanism of wave dissipation examined in this paper is its extreme sensitivity to the magnitude of the magnetic field. Increasing $B_{0}$ slightly can drastically increase $L_{z}$. That is why it seems reasonable that the waves considered here can dissipate effectively if $B_{0} \lesssim 5 \mathrm{G}$ and the wave period $\tau \gtrsim 200 \mathrm{~s}$. This conclusion does not agree with that of Gordon and Hollweg [2], who state that only surface waves with periods shorter than a few tends of seconds might heat the corona. The existence of short-period oscillations requires, however, stronger magnetic fields which in turm means extremely long dissipation lengths. If one accepts Athay's [21] estimation that for quiet coronal regions the magnetic field should be a few gauss, then the long-period (say 5-minute) oscillations can be effectively dissipated. For lower magnetic 
fields the pseudosurface mode quite poissibly becomes a pure surface one (i.e. the Bessel functions $J_{0}, J_{1}$ are replaced by the modified ones $\left.I_{0}, I_{1}[7]\right)$, but in a thin cylinder limit $(k a \ll 1)$ there is practically no difference between the two kinds of waves. In both cases the wave field inside the cylinder is almost constant and decays in radial direction outside the column.

[1] E. R. Priest, Solar Magnetohydrodynamics, D. Reidel, Dordrecht 1982, Chapter 6.

[2] B. E. Gordon and J. V. Hollweg, Astrophys. J. 266, 373 (1983).

[3] B. Leroy and St. J. Schwarz, Astronom. Astrophys. 112, 84 (1982).

[4] St. J. Schwarz and B. Leroy, Astronom. Astrophys. 112, 93 (1982).

[5] B. Roberts, P. M. Edwin, and A. O. Benz, Astrophys. J. 279, 857 (1984).

[6] J. V. Hollweg, J. Geophys. Res. 90, 7620 (1985).

[7] P. M. Edwin and B. Roberts, Solar Phys. 88, 179 (1983)

[8] I. Zhelyazkov and P. Nenovski, Physica 63, 182 (1973).

[9] A. E. H. Love, Some Problems of Geodynamics, Cambridge University Press, Cambridge 1911, Chapter 11.

[10] S. I. Braginskii, in Voprossy Teorii Plazmy (Ed. M. A. Leontovich) Vol. 1, Gosatomizdat, Moscow 1963.

[11] R. W. P. McWhirter, P. C. Thoneman, and R. Wilson, Astronom. Astrophys. 40, 63 (1975).

[12] D. P. Cox and W. H. Tucker, Astrophys. J. 157, 1157 (1969).

\section{Acknowledgements}

It is a pleasure to thank Vladimir Dermendjiev for very useful discussions of solar chromospheric and coronal conditions, Petko Nenovski for some valuable remarks, and Pat Edwin for her comments on an earlier draft of this paper.

[13] T. J. M. Boyd and J. T. Sanderson, Plasma Dynamics, Nelson, London 1969, Chapter 8.

[14] R. G. Giovanelli, Solar Phys. 44, 299 (1975).

[15] D. G. Wentzel, Astronom. Astrophys. 76, 20 (1979).

[16] J. E. Vernazza, E. H. Avrett, and R. Loeser, Astrophys. J. Suppl. 45, 635 (1981).

[17] V. M. Sobolev, Solnechnye Dannye, Bull. No. 6, 112 (1982).

[18] S. Koutchmy, Y. D. Žugžda, and V. Ločans, Astronom Astrophys. 120, 185 (1983).

[19] V. N. Dermendjiev, M. Rybanský, V. Rušin, G. T Buyukliev, and E. B. Zlateva, Contributions to the Astronomical Observatory Skalnaté Pleso 15, 429 (1986).

[20] M. Rybanský, V. Rušin, V. N. Dermendjiev, and G. T. Buyukliev, Bull. Astron. Inst. Czechosl. 37, 253 (1986)

[21] R. G. Athay, in Physics of the Sun, Vol. II, The Solar Atmosphere (eds. P. A. Sturrock, T. E. Holzer, D. M Mihalas, and R. K. Ulrich), D. Reidel, Dordrecht 1986, Chapter 9.

[22] E. R. Priest, Rep. Prog. Phys. 48, 955 (1985).

[23] P. S. Cally, Astronom. Astrophys. 136, 121 (1984). 